\title{
Substitution de Ciment pour Béton par des Pourcentages de Poudre de Marbre
}

\author{
Abdellah HAOUAT and Omar AZZOUZ \\ ${ }^{1}$ Ingénieur doctorant en génie civil. Faculté des Sciences Oujda. Spécialité : Géo matériaux. Laboratoire des géosciences appliqués \\ ${ }^{2}$ Professeur d'enseignement supérieur. Faculté des Sciences Oujda. Spécialité : Géo matériaux. Laboratoire des géosciences \\ appliqués
}

\begin{abstract}
Résumé : Le but de cette étude est la valorisation des résidus minéraux en tant que substitution partielle du ciment qui entre dans la fabrication du béton. Il s'inscrit dans une démarche de développement durable. Il regroupe les résultats obtenus expérimentalement. L'utilisation des poudres de marbre en remplacement partiel du ciment Portland dans le béton permet de réduire les émissions de gaz à effet de serre et génère un béton ayant un impact moindre sur l'environnement. En mettant à profit diverses techniques expérimentales, une attention particulière est accordée au comportement de la poudre de marbre finement broyée associée au ciment Portland avec addition de calcaire. Cette étude a confirmé l'amélioration des propriétés physiques des bétons avec l'ajout de poudre de marbre, ce qui laisse augurer de bonnes perspectives pour son utilisation comme substituant de ciment.
\end{abstract}

Mots-clés : béton, développement durable, ciment, l'environnement, poudre.

\begin{abstract}
The aim of this study is the valorization of mineral residues as a partial substitution of the cement which goes into the manufacture of concrete. It is part of a sustainable development approach. It brings together the results obtained experimentally. The use of marble powders as a partial replacement for Portland cement in concrete reduces greenhouse gas emissions and generates concrete with less impact on the environment. By taking advantage of various experimental techniques, special attention is paid to the behavior of finely ground marble powder associated with Portland cement with the addition of limestone. This study confirmed the improvement in the physical properties of concrete with the addition of marble powder, which bodes well for its use as a cement substitute.
\end{abstract}

Keywords: concrete, sustainable development, cement, the environment, powder.

\section{1- Le Béton}

\section{1-1 Définitions}

Le béton est utilisé plus que tout autre matériau synthétique dans le monde. Généralement le béton est utilisé pour faire des trottoirs, des tuyaux, des structures architecturales, des fondations, des autoroutes / routes, ponts / viaducs, structures de stationnement, murs de brique / bloc et semelles de fondation pour portes, clôtures et poteaux. C'est pour ces raisons qu'une nouvelle enquête sur la réutilisation de la poudre de marbre dans la production de béton semblait être justifiée.

Le béton est un mélange de ciment, de sable (agrégats fins), de petites pierres ou de gravier (gros granulats), de l'eau et d'autres matériaux à base de ciment, tels que cendres volantes et ciment de laitier, et adjuvants chimiques. Le ciment 
couramment utilisé est celui de Portland et généralement le les granulats sont distingués en granulats grossiers, faits de graviers ou concassés des roches telles que le calcaire ou le granit et des agrégats fins tels que le sable. À travers un produit chimique réaction appelée hydratation, la pâte durcit et gagne en force pour former la masse rocheuse connu sous le nom de béton.

\section{2- Matériaux}

Pour cette étude, les matériaux mélangés ont été dérivés de la combinaison de quatre composants et eau : ciment (CEM), agrégats grossiers (AC), agrégats fins (FA) et poudre de marbre. Premièrement, ont été effectuées des déterminations physiques, minéralogiques et chimiques afin de caractériser la poudre de marbre. Deuxièmement, des spécimens de béton ont été préparés en mélangeant les composants avec de la poudre de marbre avec des proportions différentes.

\section{3- Phase expérimentale}

La conception du mélange dépend du type de structure en construction, de la manière dont le béton sera mélangé et livré, et comment il sera placé pour former cette structure. Mélanger le béton correctement est vital pour des fondations durables, afin de créer un mélange de béton approprié.

Le caractère du béton est déterminé par la qualité de la pâte. La dureté de la pâte, à son tour, dépend du rapport eau / ciment. Le rapport eau / ciment c'est le poids de l'eau de mélange divisé par le poids du ciment. Le béton de haute qualité c'est en abaissant autant que possible le rapport eau-ciment sans sacrifier la maniabilité du béton frais.

En général, en utilisant moins d'eau, on obtient une qualité supérieure du béton à condition que le béton soit correctement mis en place, consolidé et durci.

Le béton se solidifie et durcit après avoir été mélangé avec de l'eau et mis en place à cause d'un processus chimique connu sous le nom d'hydratation.

L'eau réagit avec le ciment, qui lie les autres composants ensemble, pour finalement créer un matériau ressemblant à de la pierre.

Le calcul théorique du dosage doit toujours être ajouté avec un test expérimental pour vérifier que les exigences étaient remplies, si elles n'étaient pas atteintes, on procéderait à corriger le dosage ou, si nécessaire, changer de méthode.

Ce projet était concentré sur un mélange général.

Pour l'étude, nous avons choisi une résistance de $30 \mathrm{MPa}$ et un rapport eau-ciment associé de 0,55. Pour un module de finesse de sable de calculé de 2,7.

\section{3-1. Mélanges du béton}

Les mélanges de béton ont été conçus en supposant un bon degré de contrôle de la qualité et une condition d'exposition. Les mélanges sont conçus pour CMI_0 CMI_5 CMI_10 CMI_15 CMII_0 CMII_5 CMII_10 CMII_15. Tableau 3-1

Pour le premier calcul de mélange calculé, nous avons étudié plusieurs recherches scientifiques et appliqué les mêmes procédures et mêmes proportions (Güneyisi, E., et al., 2008).

Pour le second, on a utilisé la méthode ACI (Fernández, M. 2007). La méthode Américaine de conception des mélanges du béton (ACI) est l'une des nombreuses méthodes de conception des mélanges de béton de base. 
Cette méthode consiste à choisir une résistance minimale pour le béton d'essai (Fernández, 2007), en fixant une valeur de résistance, le rapport eau / ciment étant associé à cette résistance.

Pour l'étude, nous avons choisi une résistance de $30 \mathrm{MPa}$ et un rapport eau-ciment associé de 0,55. Pour un module de finesse de 2,7 .

Tableau 3-1 : proportion de mélanges des deux conceptions de mélanges [kg]

\begin{tabular}{|c|c|c|c|c|c|c|}
\hline \multicolumn{7}{|c|}{ MIX-DESIGN I } \\
\hline & W/(CEM+MD) & CEM & FA & CA & MD & WATER \\
\hline CMI_0 & 0.5 & 8.3 & 12.5 & 25 & 0 & 4.2 \\
\hline CMI_5 & 0.5 & 7.9 & 12.5 & 25 & 0.4 & 4.2 \\
\hline CMI_10 & 0.5 & 7.5 & 12.5 & 25 & 0.8 & 4.2 \\
\hline CMI_15 & 0.5 & 6.7 & 12.5 & 25 & 1.6 & 4.2 \\
\hline \multicolumn{7}{|c|}{} \\
\hline
\end{tabular}

Les bétons de contrôle (sans poudre de marbre) pour les mélanges ont été réalisés sans incorporer de poudre marbre.

Ainsi, les éprouvettes ont été préparées au laboratoire par mélange direct de ciment (CEM), agrégats grossiers (AC), agrégats fins (AF) et ajout de poudre de marbre (MD) en différentes proportions (en poids) et d'eau (Figure 3-1). Le ciment est mélangé avec du sable et de l'eau pour former du béton. La quantité exacte d'ingrédients du béton a été pesée et mélangée.

Le ciment a été remplacé par la poudre de marbre à différents pourcentages en poids $(0 \% ; 5 \% ; 10 \% ; 15 \%)$ pour préparer les mélanges de béton, fixant le rapport eau sur CEM + PD à une valeur de 0,5 (en poids) pour le mélange.

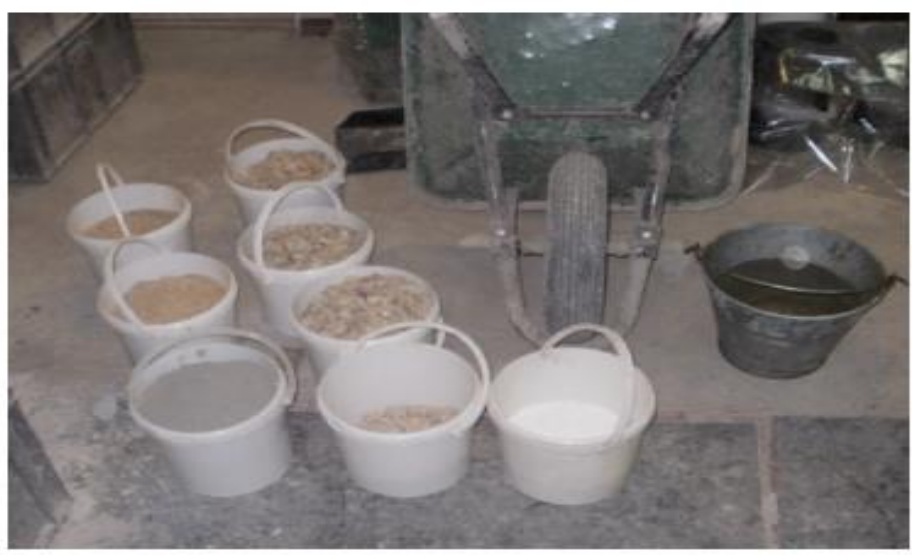

FIGURE 3-1COMPOSANTS DU BETON PRETS A ETRE MELANGES. 

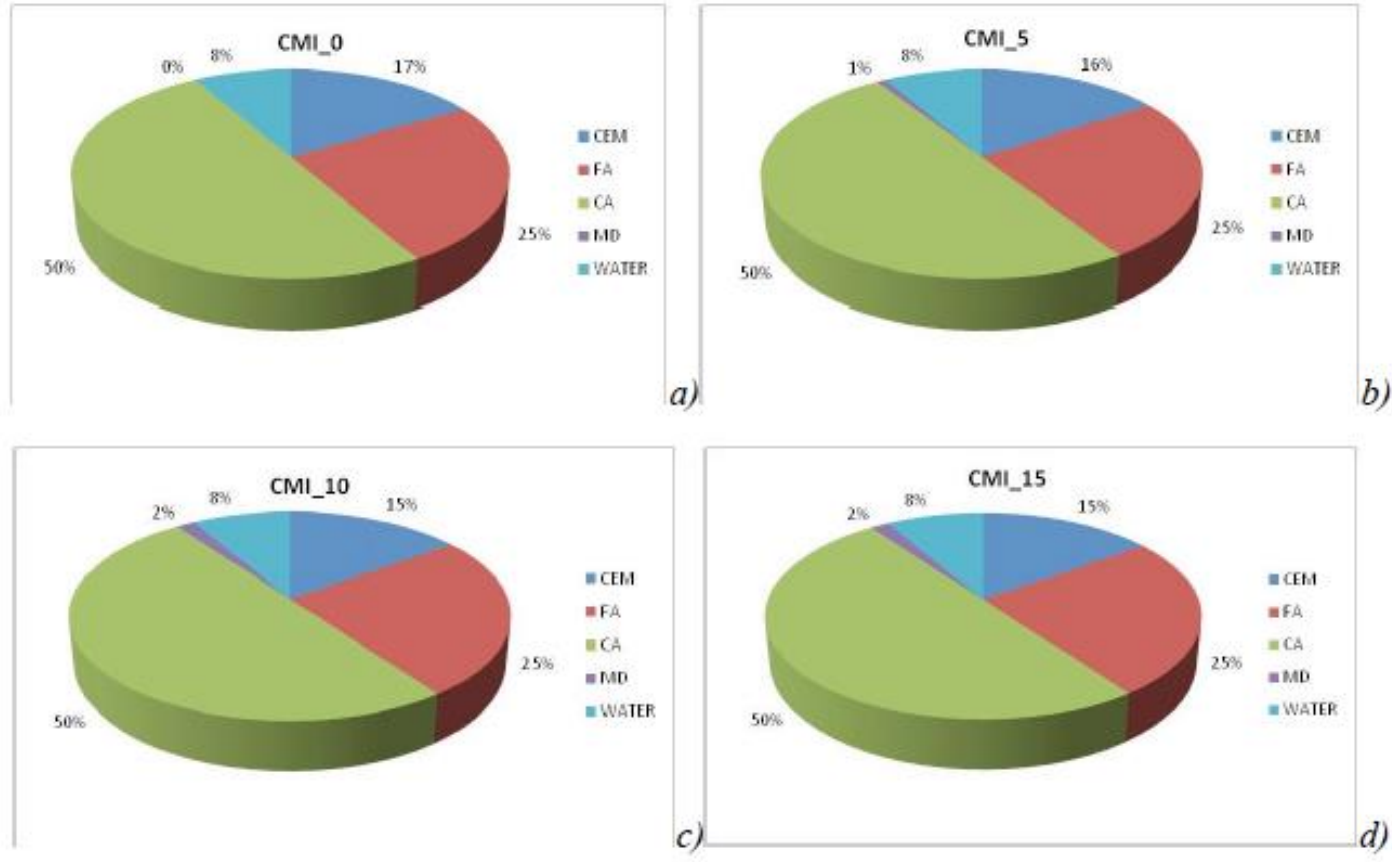

Figure 3-2: Diagrammes à secteurs a) CMI_0 b) CMI_5 c) CMI_10 d) CMI_15
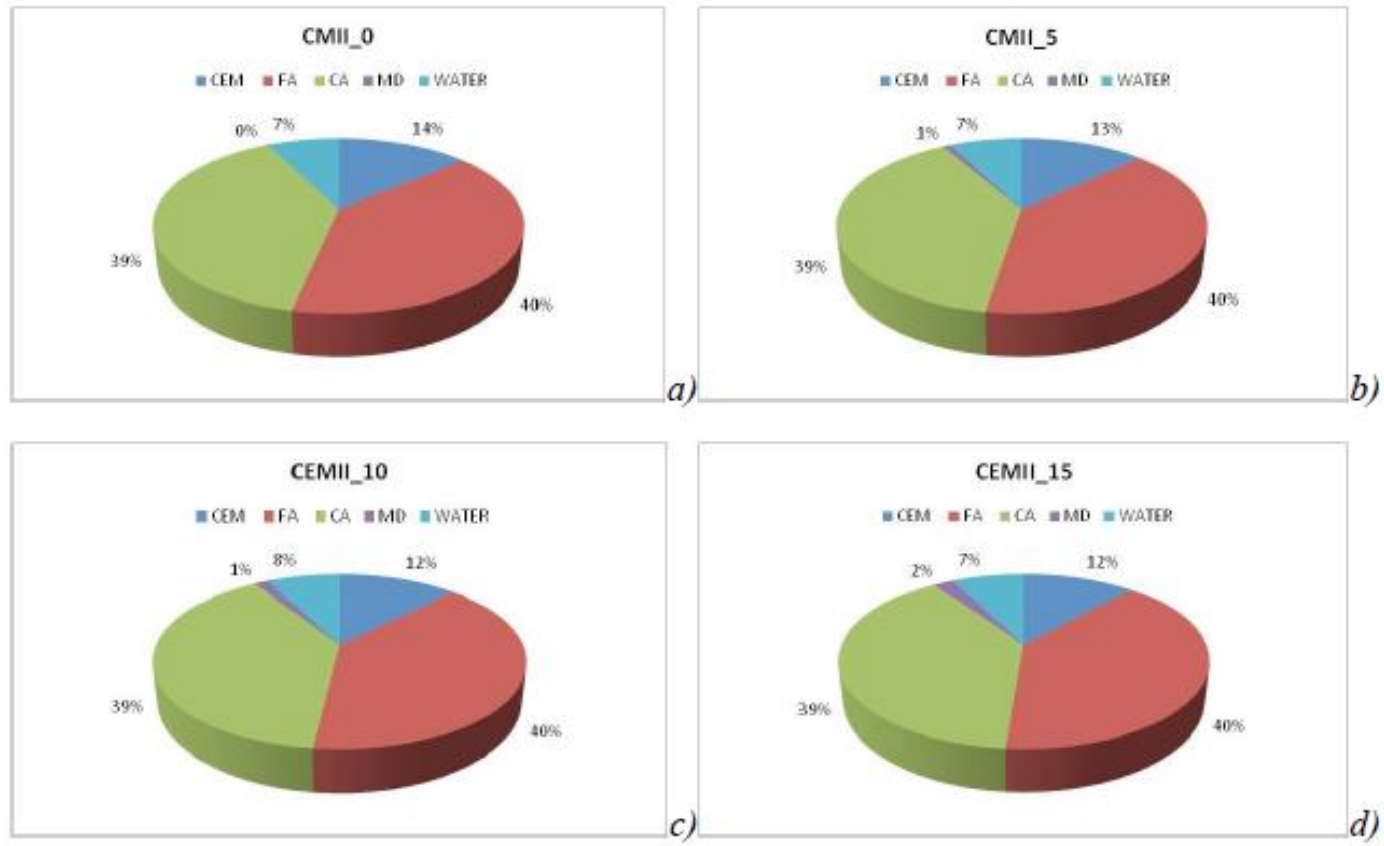

Figure 3-3: Diagrammes à secteurs a) CMII_0 b) CMII_5 c) CMII_10 d) CMII_15

\subsection{Durcissement}

Le processus de durcissement développe des propriétés physiques et chimiques. Parmi d'autres qualités, résistance mécanique, faible perméabilité à l'humidité et stabilité chimique et volumétrique. 
Pendant le durcissement, une plus grande quantité de ciment réagit avec l'eau résiduelle (hydratation). Le ciment pâte durcit avec le temps, durcissant initialement et devenant rigide bien que très faible, et gagne en durcissement dans les jours et les semaines suivants.

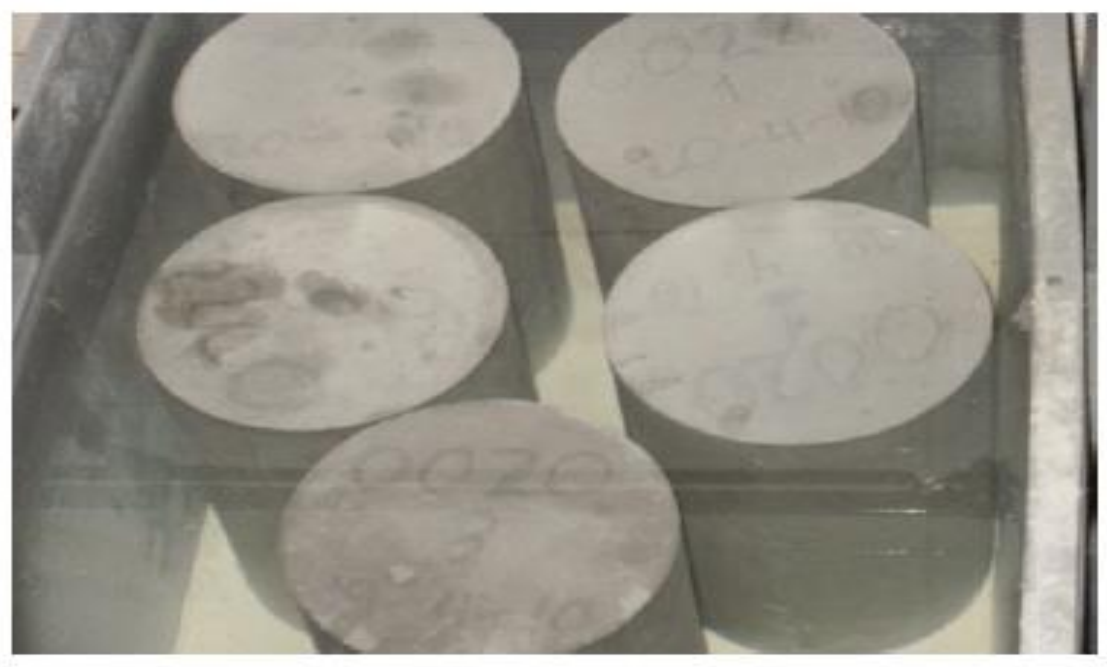

Figure3-4 : durcissement des échantillons.

\section{3-4 Test}

\section{3-4-1 Essais de résistance à la compression}

Les vingt-quatre échantillons de béton ont été étudiés sur une période de 28 jours afin de déterminer l'effet de l'addition de poudre de marbre. Les résistances à la compression ont été évaluées par rapport aux pourcentages de poudres résiduaires remplacées par du ciment.

Les tests ont été déterminés à l'aide d'une machine de test en compression (SDE: Sistemas de Ensayo, modèle: CME300 / SDC, 1998) d'une capacité maximale de $2500 \mathrm{kN}$ et ont été réalisée en laboratoire LPEE. Les souches ont été mesurées sur une base de $150 \mathrm{~mm}$.

Toutes les surfaces des roulements de la machine d'essai ont été nettoyées et toutes les particules avec matière étrangère provenant des surfaces de l'échantillon qui était en contact avec les plateaux. L'humidité en excès a été essuyée de la surface de l'échantillon avant d'être placée dans la machine d'essai.

Le spécimen était centré par rapport à la plaque inférieure avec une précision de $1 \%$ de diamètre désigné des éprouvettes cylindriques. Le spécimen a été placé dans la machine et préchargé avec une vitesse de charge constante dans la plage de $0,6 \pm 0,2 \mathrm{MPa} / \mathrm{s}$.

Lorsque l'échantillon échoue, on lit la charge maximale supportée par l'échantillon et l'enregistrée.

Nettoyez la machine après utilisation. La procédure ci-dessus a été répétée pour chaque cylindre afin de déterminer la résistance du béton. 

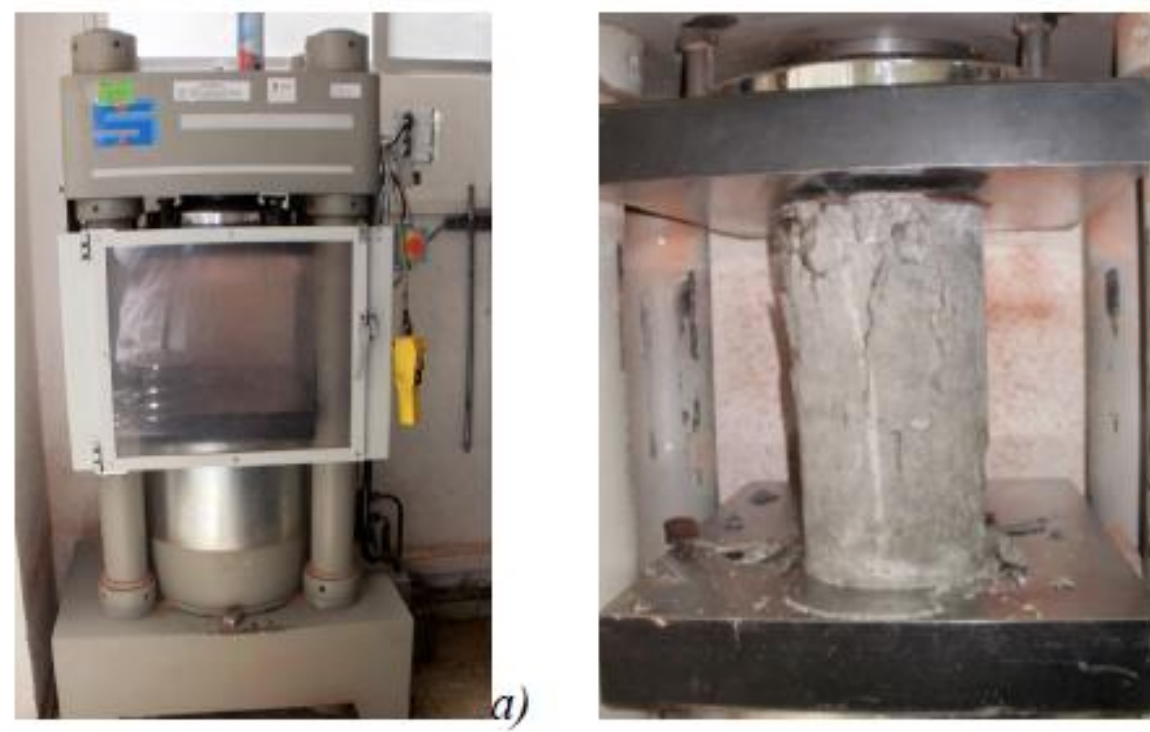

b)

Figure 3-5 : a) Machine b) essai de compression.

\section{3-4-2 Expression des résultats}

La résistance à la compression est donnée par l'équation 3-1 :

$\mathrm{f} c=\mathrm{F} / \mathrm{A} \mathrm{c}(3.1)$

Où

fc est la résistance à la compression, en $\mathrm{MPa}\left(\mathrm{N} / \mathrm{mm}^{2}\right)$;

$\mathrm{F}$ est la charge maximale à la rupture, en $\mathrm{N}$;

Ac est l'aire de la section de l'éprouvette sur laquelle agit la force de compression, calculé à partir de la taille spécifiée du spécimen (voir UNI EN 12390-1) ou à partir les mesures sur le spécimen si elles sont testées conformément à l'annexe B de la norme UNI EN 12390- 3, en mm2.

La résistance à la compression a été exprimée à $0,1 \mathrm{MPa}\left(\mathrm{N} / \mathrm{mm}^{2}\right)$ près.

Les résultats des tests effectués dans le laboratoire LPEE pour la résistance à la compression ont été listés dans tableaux et la figure ci-dessous (tableaux 3-2 et 3-3; Figure 3-6).

Tableau 3-2 : Données d'essai de compression pour la conception Mélange I [MPa]

\begin{tabular}{|c|c|c|c|c|}
\hline Mix-design I & Specimen 1 & Specimen 2 & Specimen 3 & Average value \\
\hline CMI_0 & 41.05 & 38.85 & 37.44 & 39.12 \\
\hline CMI_5 & 41.29 & 39.28 & 38.56 & 39.71 \\
\hline CMI_10 & 32.62 & 35.45 & 35.42 & 34.50 \\
\hline CMI_15 & 33.45 & 27.90 & 31.43 & 30.93 \\
\hline
\end{tabular}

Source : propre 
HAOUAT et al.: Substitution de Ciment pour Béton par des Pourcentages de Poudre de Marbre

Tableau 3-3: Données d'essai de compression pour la conception Mélange II [MPa]

\begin{tabular}{|c|c|c|c|c|}
\hline Mix-design II & Specimen 1 & Specimen 2 & Specimen 3 & Average value \\
\hline CMII_0 & 28.74 & 27.77 & 27.88 & 28.13 \\
\hline CMII 5 & 27.46 & 27.61 & 27.18 & 27.41 \\
\hline CMII_10 & 23.69 & 24.75 & 23.78 & 24.07 \\
\hline CMII_15 & 19.07 & 18.98 & 19.16 & 19.07 \\
\hline
\end{tabular}

Source : propre

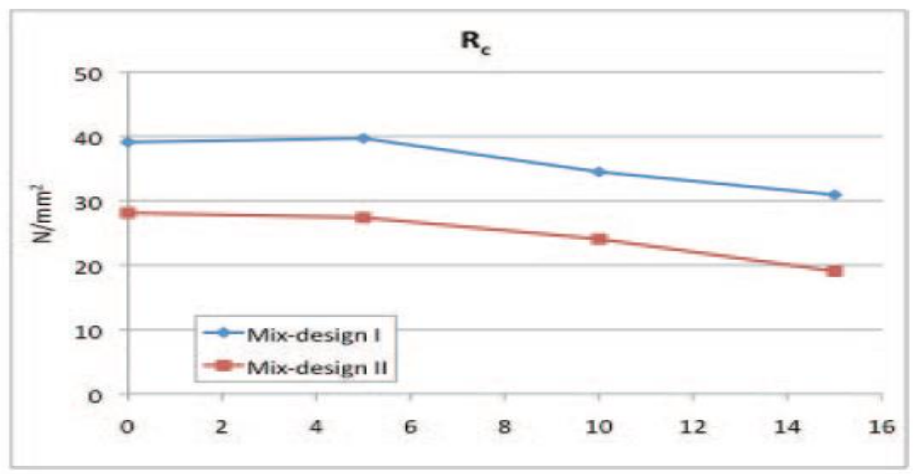

Figure 3-6: Courbes de résistance à la compression pour les deux conceptions de mélanges.

\section{4-3 Résultats et discussions}

Tous les résultats obtenus pour les tests de compression sont acceptables. Les caractéristiques étaient influencées par les pourcentages de poudre.

Comme le montrent les tableaux 3-2 et 3-3 et la figure 3-6, la valeur maximale de compression était observée pour les échantillons contenant $5 \%$ de poudre. Dans le cas de $10 \%$ de remplacement la résistance à la compression a diminué et plus dans le cas de 15\%. Donc, il a été observé dans Tableau 3-2, il y avait une réduction de la résistance à la compression avec l'augmentation de poudre de marbre.

\section{5- Conclusion}

Les possibilités techniques de fabrication de bétons contenant de la poudre de marbre ont été étudiées avec des résultats positifs. Ainsi, il peut remplacer partiellement le ciment, dans le but de minimiser la production et l'élimination des déchets ainsi que la production de revenus pour les entreprises. En fait le ciment est le composant de béton le plus chère, par conséquent, si nous pouvons remplacer le ciment, nous pouvons obtenir un revenu économique.

En termes de performances mécaniques, substitution de $5 \%$ du ciment par de la poudre de marbre fournie une résistance à la compression maximale, après 28 jours de durcissement, mais tous les résultats sont acceptables.

Le remplacement du ciment par 5\% de poudre de marbre donne un excellent résultat en aspect résistance et qualité. Les résultats ont montré que le mélange CMI_5 induisait plus de résistance à la compression, après 28 jours de 
durcissement. L'augmentation de la teneur en poudre de marbre de plus de 5\% affecte la résistance à la compression du béton.

Les résultats des tests montrent ensuite que ces poudres de marbre sont capables d'améliorer les processus de durcissement.

Les résultats présentés et discutés au cours de ce travail permettent de tirer les conclusions que la résistance à la compression du béton d'essai est légèrement supérieure à celle du béton traditionnel.

L'échantillon avec les 5\% de remplacement et que le tonnage élevé de matières premières consommées dans la fabrication du béton font de leurs formulations des matrices appropriées pour incorporer ce type de matériau remplaçant les ressources naturelles non renouvelables.

Par conséquent, les résultats de cette étude fournissent une forte recommandation pour l'utilisation du poudre marbre.

L'ajout de poudre de marbre dans la fabrication du béton donne un résultat final encourageant.

\section{Bibliographies}

[1] Almeida N., Branco F., Santos J.R., (2007). Recycling of stone slurry in industrial activities: Application to concrete misture. Building and Environment 42(2007): 810 - 819.

[2] Binici, H., Kaplan, H., Y1lmaz, S., 2007, "Influence of marble and limestone dusts as additives on some mechanical properties of concrete".In: ScientificResearch and Essay Vol.2 (9), 372-379, September 2007.

[3] Bontoux, L., Leone, F., 1997, “The Legal Definition of Waste and its Impact on Waste Management in Europe”. Office for Official Publications of the European Communities, EUR 17716 EN, November, Luxembourg.

[4] European Council Decision (2001/573/EC), Council Decision of 23 July 2001 amending Council Decision 2000/532/EC as regards the list of wastes, Official Journal of the European Communities: L 203/18, Brussels, July 2001

[5] Gertsakis, J., Lewis, H., 2003, “Sustainability and the Waste Management Hierarchy”.A discussion paper for EcoRecycle Victoria, RMIT University, Melbourne

[6] Marras, G., Careddu, N., Internicola, C., Siotto, G., 2010. "Recovery and reuse of marble powder by-product”. In: Proceedings of the Global Stone Congress 2010. 02 - 05 March 2010, Alicante (Spain).

[7] OECD (1998) Waste Management Policy Group.Final Guidance Document for Distinguishing Waste from Non-waste. ENV/EPOC/WMP(8)1/REV1. Paris, 23-24 April 1998.

[8] OSNET Editions vol. 7, “Optimising quarrying techniques and practices”, edited by N. Terezopoulos, I. Paspaliaris, Athens 2004.

[9] OSNET Editions vol. 12, "Environmental friendly practices for natural stone exploitation”, edited by A. Dieb, N. Bonito, I. Paspaliaris, Athens 2004.

[10] Pongracz, E., 2002, "Re-defining the concepts of waste and waste management - Evolving the theory of waste management", Oulu University Press, ISBN 951-42-6821-0.

[11] Poncrácz, E., Philips, PS., Keiski, R1., 2004, "From waste minimization to resources use optimization: Definitions and legislative background”. In: Pongrácz E. (ed.) Proceedings of the Waste Minimisation and Resources Use Optimisation Conference. June 10th 2004, University of Oulu.Oulu University Press.

[12] "Report on Special Waste Management in Sardinia - Analysis of MUD 2005 data" published by the Environmental Protection Department of the Autonomous Region of Sardinia (April 2007).

[13] Segadães, A.M., Carvalho, M.A., Acchar, W., Appl. Clay Sci. 30 (2005) 42-52.

[14] SiddarthPareek, 2001. "Gainful utilization of Marble waste. An effort towards protection of ecology and environment", and http.//www.cdos- india.com/papers. 